\title{
Uso académico de redes sociales: análisis comparativo entre estudiantes de Ciencias y de Letras
}

\author{
Sergio Roses \\ Universidad de Málaga (España) \\ sergioroses@uma.es \\ Marisol Gómez Aguilar \\ Universidad de Málaga (España) \\ marisol@uma.es \\ Pedro FARIAS \\ Universidad de Málaga (España) \\ farias@uma.es
}

\section{Resumen}

El artículo compara el uso académico de las redes sociales en universitarios de distintas áreas de conocimiento. A través de un estudio empírico, se analizan las diferencias entre estudiantes de "ciencias" (titulaciones de Ciencias Experimentales, Ciencias de la Salud, Ingenierías) y estudiantes de "letras" (titulaciones de Humanidades y Ciencias Sociales y Jurídicas). Los estudiantes de ciencias presentaban actitudes menos favorables a la utilización de las redes sociales en la docencia. Asimismo, usaban menos las redes sociales en el ámbito académico que sus compañeros de letras.

Palabras clave: Redes Sociales; Innovación educativa; EEES; Innovación didáctica; Aprendizaje; Estudiantes universitarios.

\section{The Academic Use of Social Networks: A Comparative Analysis between Science Students and Social Science Students}

\begin{abstract}
This paper examines the academic use made of the social networks by university students according the area of knowledge of their Bachelor. An empirical study was conducted to observe the differences between "Science Students" (Bachelors on Experimental Sciences, Health or Engineering) and "Social Science students" (Bachelors on Arts, Humanities, Social Sciences and Law). Science students showed less favorable attitudes to the use of social media in teaching. Their use of social network for academic purposes was lower than the Social Science Students' use too.
\end{abstract}

Key Words: Social Networks; Educational Innovation; EHEA; Teaching innovation; Learning; University Students.

\section{Referencia normalizada:}

Roses, S.; Gómez Aguilar, M. y Farias, P. (2013) Uso académico de redes sociales: análisis comparativo entre estudiantes de Ciencias y de Letras. Historia y Comunicación Social. Vol. 18. N ${ }^{\circ}$ Especial Diciembre Págs. 667-678. 
Sumario: 1. Introducción. 1.1. Redes sociales y docencia universitaria. 1.2. Estudios empíricos y experiencias docentes. 2. Metodología. 3. Resultados. 3.1. Uso genérico. 3.2. Motivaciones y actitudes. 3.3. Uso académico. 4. Conclusiones. 5. Referencias bibliográficas.

\section{Introducción}

La creciente relevancia de las redes sociales y las comunidades virtuales en el ámbito comunicativo ha supuesto la aparición de novedosas formas de interacción entre individuos, primando un modelo de comunicación horizontal que posibilita compartir conocimiento e información de manera ágil y barata. Así, el desarrollo del software social se posiciona como una de las grandes victorias de la Sociedad del Conocimiento. Precisamente, su máximo exponente, las redes sociales, ha atraído la atención de distintos ámbitos de la realidad social, porque el 93\% de los internautas tiene al menos una cuenta activa en redes sociales (The Cocktail Analysis, 2013). Los jóvenes adultos son el grupo social que presenta a priori una mayor pericia e intensidad en su uso: comparten música, mensajes, imágenes, comentarios; interactúan entre ellos y con desconocidos; discuten u organizan eventos, entre otras posibilidades. Buckinghan y Bautista (2013) plantean que la juventud hace tiempo que usa los recursos disponibles en la red para algo más que buscar información; actualmente, se valen de las redes para recrear un foro donde edificar su identidad y administrar sus relaciones sociales. Explican que los medios sociales se han convertido en un recurso para los jóvenes que intentan reforzar su capital social, crear comunidad y aprender. Precisamente, este estudio se centra en la utilidad que los jóvenes universitarios pudieran estar dando a las redes sociales en el ámbito académico. A partir de un estudio empírico, esta investigación trata de aportar pruebas sobre la existencia de diferencias en el uso académico de las redes sociales entre los estudiantes de titulaciones 'de ciencias' y 'de letras'. Las disimilitudes referidas al uso de redes deberían suscitar estrategias y prácticas docentes alternativas según el colectivo de alumnos a quien se dirijan.

\subsection{Redes sociales y docencia universitaria}

No es de extrañar que el sector de la educación, especialmente el universitario, haya visto en las redes una oportunidad para mejorar su desempeño (Cobo y Romaní, 2007; Castañeda, 2003). En esta línea, Meso, Pérez y Mendiguren (2011) plantean que la rápida implantación y consolidación de las redes sociales evidencian la necesidad de utilizarlas como plataforma para la docencia, ya que aportan grandes posibilidades a la formación y se proyectan como un recurso significativo para el aprendizaje.

El énfasis en el aprendizaje autónomo y colaborativo que ha supuesto el cambio metodológico propugnado por el Espacio Europeo de Educación Superior (MEC, 2005) ha favorecido un contexto muy propicio para el uso de las redes sociales. A priori podrían alcanzarse objetivos como el aumento de la implicación del estudiante, la incorporación de metodologías activas y el trabajo en equipo y la participación del 
docente como gestor de entornos estimulantes de aprendizaje. De acuerdo a la literatura, las redes sociales pueden considerarse instrumentos adecuados para la docencia universitaria por varios motivos:

Fomentan el autoaprendizaje, el trabajo en equipo (con el que se obtienen valores como la solidaridad, el compromiso y una disposición positiva), la retroalimentación, el aprendizaje constructivista y el contacto con expertos. Las redes se entienden como herramientas de gestión intelectual para el aprendizaje continuado durante la vida (Castañeda, 2010: 95; Peña, Pérez y Rondon, 2010; Duart y Sangrà, 2005).

Dotan al alumno de un papel más activo, fomentando la interacción entre alumnos y docentes y rompiendo las barreras espacio-temporales (actualización permanente) (Alemañy, 2009; Castañeda, 2010; Meso, Pérez y Mendiguren, 2011).

Proveen al estudiante de un entorno creativo con múltiples herramientas y materiales (sonidos, imágenes, vídeos, archivos). Crean un espacio paralelo al aula física donde los estudiantes pueden surtirse de gran cantidad de información y recursos más allá de la programación establecida (Alemañy, 2009; Meso, Pérez y Mendiguren, 2011).

Cambian las estrategias de enseñanza-aprendizaje (Kieslinger y Ehms, 2010). El rol del profesor cambia. De suministrador de conocimientos, se transforma en guía o gestor, diseñador y elaborador de entornos sociales, virtuales e interactivos. Es decir, las responsabilidades y funciones abandonan el modelo formal y evolucionan hacia una modelo diferente.

A pesar de todo lo dicho, la "apropiación" de la tecnología por parte del alumnado suele preceder a la del profesorado; por tanto, al margen de que algunos docentes universitarios estén utilizando las redes sociales como recurso educativo, es razonable pensar que los estudiantes motu proprio están realizan algún tipo de uso académico no dirigido de las redes, tal y como algunos estudios han demostrado (Gómez, Roses y Farias, 2012). Asimismo, aunque se presuma buena disposición entre el alumnado, la actitud podría variar según el tipo de titulación o de área de conocimiento de los estudiantes.

\subsection{Estudios empíricos y experiencias docentes}

De acuerdo a las bondades sobre el proceso educativo que la literatura atribuye a las redes sociales, durante los últimos años, se han iniciado proyectos basados en la utilización de redes sociales en el ámbito docente. Encontramos experiencias en el área de conocimiento de las Ciencias Sociales y las Humanidades (e.g,. Espuny et al., 2011; López y Tascón, 2013; Fernández, Revuelta y Sosa, 2012) y, sobresale la explotación que de las redes están haciendo los profesores del área de Comunicación y Periodismo; probablemente, porque son directamente parte natural de su objeto de estudio (e.g. Túñez y Sixto, 2012; Mendiguren, Meso y Pérez, 2012; Subires y Olmedo, 2013). El diseño de acciones que vinculan la utilización de las redes en las aulas también ha proliferado entre profesores universitarios "de ciencias" (e.g. De 
Miguel et al., 2012), siendo habitual incorporar tareas de evaluación de la satisfacción de los estudiantes (e.g. Garrigós et al., 2010). En este sentido, varias experiencias han alcanzado un alto grado de aceptación entre sus estudiantes (Martínez; Corzana y Millán, 2013), cosechando actitudes que refrendan la utilización de redes sociales en su actividad docente (Avendaño et al., 2012).

El análisis global de estos trabajos empíricos y experiencias docentes indica que la mayor parte se diseña y aplica en el contexto de una titulación específica. No hallamos estudios empíricos que comparen los mismos presupuestos en estudiantes de distintas áreas. Por tanto, los resultados de los estudios mencionados anteriormente podrían adolecer de un déficit de validez externa cuando las técnicas docentes y las valoraciones sobre los efectos del uso de redes sobre el aprendizaje se extrapolan a todo tipo de estudiante universitario.

Vista la deficiencia de estudios comparativos en la literatura, el objetivo de la investigación es estudiar las disimilitudes en el uso académico de redes sociales entre estudiantes de las titulaciones popularmente denominadas "de ciencias" (Ciencias Experimentales, Ciencias de la Salud, Ingenierías) y estudiantes de titulaciones "de letras" (Humanidades y Ciencias Sociales y Jurídicas). Puesto que no existen trabajos previos que susciten el establecimiento de una hipótesis de partida, se estableció la siguiente pregunta de investigación:

P1.: ¿Existen diferencias significativas en las actitudes y uso académico de las redes sociales entre los estudiantes "de ciencias" y "de letras"?

\section{Metodología}

La investigación se construyó desde un planteamiento cuantitativo. Se usó una encuesta suministrada en papel en 2011 con la ayuda de un grupo de voluntarios de investigación, previamente entrenados. El cuestionario recogía preguntas, agrupadas por temáticas: uso genérico de redes sociales, actitudes hacia el uso de redes sociales, uso específico de las redes sociales con fines académicos y actitudes hacia el uso académico de las redes sociales (véase su operacionalización en Gómez Aguilar, Roses y Farias, 2012). La población la constituyeron los estudiantes de 1er o 2 do ciclo de la Universidad de Málaga (UMA), con un tamaño de 32.464 estudiantes, según el último censo disponible. Se usó un muestreo de tipo probabilístico por conglomerados, correspondientes a cinco ramas de enseñanza por cada ciclo. La elección de la titulación a entrevistar dentro de cada conglomerado se hizo de forma aleatoria simple a partir de la utilización de un programa informático. Se preservó la estructura poblacional a través de la fijación de las cuotas de ciclo y ramas de enseñanza, esto es, se calculó el número proporcional de encuestas a realizar en función del peso relativo de la cuota en la estructura de la población. El tamaño de la muestra fue de 1.033 estudiantes, para un nivel de confianza del $95 \%$ y un error del $+/-3 \%$. Revisada y depurada la matriz de datos, el número total de individuos de la muestra 
que se identificó como usuario de redes sociales fue de 855 estudiantes; por tanto, en lo sucesivo, los descriptivos y porcentajes incluidos en este artículo se han calculado sobre dicha base.

Se empleó SPSS para el análisis de datos. Para evitar errores estadísticos derivados de los tamaños de los grupos, la variable "Área de conocimiento" (Ciencias Experimentales $=1$, Ciencias de la Salud $=2$, Humanidades $=3$, Ingenierías $=4$, Ciencias Sociales y Jurídicas $=5$ ) se recodificó en una variable nominal con dos categorías $($ Ciencias $=1$, Letras $=2)$. En el caso de la comparación de medias, se usó la prueba T de Student para muestras independientes. En el caso de la comparación de variables categóricas, se utilizaron tablas de contingencia, el estadístico Chi Cuadrado de Pearson y los residuos tipificados corregidos.

Las características de la muestra definitiva de usuarios de redes sociales $(\mathrm{n}=855)$ fueron las siguientes. La mayor parte de los encuestados fueron mujeres $(54,2 \%)$ y la edad media de los estudiantes ascendió a 21,43 años $(\mathrm{DT}=3,54)$. Los estudiantes de primer y segundo curso fueron los más numerosos en la muestra $(69,4 \%)$. Debido a la actual oferta académica de la Universidad de Málaga, el porcentaje de estudiantes de la muestra que pertenecía a alguna titulación "de letras" fue del $63,2 \%$ mientras que los estudiantes de carreras "de ciencias" supusieron el 36,8\%. El porcentaje de hombres fue significativamente superior entre los estudiantes "de ciencias" $(63,8 \%)$ que entre los estudiantes "de letras" $(35,4 \%)\left[\chi^{2}(1, \mathrm{~N}=855)=64.809, \mathrm{p}<0.05\right]$. Asimismo, el porcentaje de estudiantes de los dos primeros cursos era significativamente mayor entre los estudiantes "de ciencias" $(74,3 \%)$ que entre los estudiantes "de letras" $(66,5 \%)\left[\chi^{2}(1, N=855)=5,701 . p<0,05\right]$. No se hallaron diferencias en la media de edad entre los dos grupos.

\section{Resultados}

\subsection{Uso genérico}

Los estudiantes de ambos grupos se iniciaron a la vez en el uso de las redes. Acumulaban un número similar de contactos/seguidores en el momento en que fueron encuestados y tampoco hubo diferencias en el número de redes que utilizaban. Sin embargo, los resultados evidenciaron la presencia de algunas diferencias significativas en el uso general de redes sociales entre los dos grupos estudiados. Los estudiantes "de ciencias" usaban las redes con menos frecuencia $(\mathrm{M}=7,13 . \mathrm{DT}=1,12)$ que los "de letras" $(\mathrm{M}=7,41 . \mathrm{DT}=0,86)[\mathrm{t}(534,350)=-3,890 . \mathrm{p}<0,05]$. Asimismo, la exposición diaria a las redes en minutos era significativamente menor entre los estudiantes "de ciencias" $(\mathrm{M}=80,53$. $\mathrm{DT}=56,48)$ que entre los "de letras" $(\mathrm{M}=91,91$. $\mathrm{DT}=58,40),[\mathrm{t}(804)=-2,702 . \mathrm{p}<0,05]$. Buscamos diferencias en el tiempo dedicado a las redes sociales más populares. No se hallaron tendencias desiguales en el uso de Facebook, Twitter y Linkedin. Sin embargo, los estudiantes "de ciencias" dedi- 
caban significativamente menos tiempo a Tuenti-la red social más utilizada por los estudiantes de la muestra - $(\mathrm{M}=3,47 . \mathrm{DT}=1,25)$ que los estudiantes "de letras" $(\mathrm{M}=$ $3,70$. $\mathrm{DT}=1,26),[\mathrm{t}(833)=-2,537 . \mathrm{p}<0,05]$. Respecto a los dispositivos que usan para conectarse a las redes, no hallamos diferencias significativas en la frecuencia con que se conectan por medio de tablets y móviles. Sin embargo, los estudiantes "de ciencias" utilizaban el ordenador de sobremesa con mayor frecuencia $(\mathrm{M}=3,43$. DT= $1,44)$ que los "de letras" $(\mathrm{M}=3,01$. $\mathrm{DT}=1,53),[\mathrm{t}(834)=3,882 . \mathrm{p}<0,05]$. Al contrario, los estudiantes "de letras" usaban los ordenadores portátiles con mayor asiduidad $(\mathrm{M}=3,63 . \mathrm{DT}=1,440)$ que los "de ciencias" para conectarse $(\mathrm{M}=3,25 . \mathrm{DT}=1,57)$ $[\mathrm{t}(593,417)=-3,427 . \mathrm{p}<0,05]$.

De entre 13 actividades genéricas para las que se pueden emplear las redes sociales, hallamos diferencias significativas entre grupos en el tiempo que habitualmente pasan realizando seis de ellas. La única actividad a la que los estudiantes "de ciencias" dedicaban significativamente más tiempo que los "de letras" fue "Buscar pareja o ligar" $[\mathrm{t}(525,038)=2,738$. $\mathrm{p}<0,05]$. En cambio, los estudiantes "de letras" pasaban significativamente más tiempo "informándose sobre lo que pasa en su grupo de amigos" [t(560,162) $=-3,798$. $\mathrm{p}<0,05]$, "consultando noticias" [t(830) $=-2,683$. $\mathrm{p}<$ $0,05]$, "contando lo que están haciendo en ese momento" $[\mathrm{t}(662,025)=-3,710$. $\mathrm{p}<$ $0,05]$, "compartiendo archivos" [ $\mathrm{t}(594,336)=-2,695 . \mathrm{p}<0,05]$, "comentando fotos o vídeos" $[\mathrm{t}(594,554)=-3,706$. $\mathrm{p}<0,05]$ y "curioseando o cotilleando otros perfiles" $[\mathrm{t}(837)=-2,076 . \mathrm{p}<0,05]$. Preguntamos por la frecuencia con que usaban 11 aplicaciones presentes en Facebook y/o Tuenti. Nuestro ánimo era el de identificar las herramientas con que estaban más familiarizados y así plantear actividades académicas en el futuro. Solo hubo diferencias significativas en dos de ellas. Los estudiantes "de letras" utilizaban con más frecuencia que los "de ciencias" "el muro" [t(835)= $-2,486 . \mathrm{p}<0,05]$ y "las fotos" [t(845) $=-2,517 . \mathrm{p}<0,05]$.

\subsection{Motivaciones y actitudes}

No se hallaron diferencias significativas respecto del motivo por el que utilizaban las redes sociales. Una cuarta parte de los estudiantes de cada grupo punteó a "los estudios" como una de sus motivaciones. Sin embargo, algo más de un tercio de cada uno de los grupos afirmó que "perdía tiempo de estudio para dedicarlo a las redes sociales". En este caso, tampoco se encontraron diferencias significativas entre estudiantes "de ciencias" y "de letras".

Los alumnos de titulaciones de "ciencias" presentaron actitudes más desfavorables ante el uso académico de las redes que sus compañeros de "letras". Así, el porcentaje de universitarios que valoró negativamente la posibilidad de crear un perfil de una asignatura en una red social fue significativamente superior entre los estudiantes de "ciencias" $(7,7 \%)$ que entre los "de letras" $(4,3 \%)$. Igualmente, el porcentaje de estudiantes "de ciencias" $(15,1 \%)$ "indecisos" fue estadísticamente superior al del otro grupo $(9,2 \%)\left[\chi^{2}(3, N=847)=12,799 . p<0,05\right]$. Asimismo, el porcentaje de estudiantes "de ciencias" que valoraba positivamente la posibilidad de usar para las clases una red social en vez del campus virtual de la universidad fue significativamente inferior 
$(33,7 \%)$ al de estudiantes "de letras" $(43,4 \%)$. También el porcentaje de estudiantes "de ciencias" que valoró negativamente la propuesta fue significativamente superior $(24,4 \%)$ al de sus compañeros "de letras" $(18,1 \%)\left[\chi^{2}(3, \mathrm{~N}=847)=11,015 . \mathrm{p}<0,05\right]$.

Ambos grupos expresaron, en promedio, bastante desacuerdo con que las redes sociales son un lugar donde los estudiantes pueden encontrar algún tipo apoyo académico. No obstante, la actitud de los estudiantes "de letras" fue significativamente más favorable que la de sus compañeros "de ciencias". Así ocurre con el supuesto de que "en las redes encuentran a personas que les ayudan a resolver problemas relacionados con sus estudios" [t(600,273) $=-3,131 . \mathrm{p}<0,05]$. También mostraron mayor acuerdo $(M=2,68$. DT=1,24) que sus compañeros "de ciencias" $(M=2,49$. $\mathrm{DT}=1,29)$ con que "encuentran ayuda cuando preparan los exámenes" [ $\mathrm{t}(623,563)$ $=-2,028 . p<0,05]$, "ayuda para resolver dudas sobre las asignaturas" [ $[\mathrm{t}(620,431)=$ $-3,272$. $\mathrm{p}<0,05]$, con que "encuentran a personas que les proporcionan materiales útiles para sus estudios" $[\mathrm{t}(839)=-2,890$. $\mathrm{p}<0,05]$ y "con las que pueden compartir el trabajo de clase" $[\mathrm{t}(603,107)=-3,723 . \mathrm{p}<0,05]$.

\subsection{Uso académico}

Los resultados indican que existen algunas diferencias significativas en el uso académico de las redes sociales entre los alumnos "de ciencias" y los "de letras". Los dos grupos se conectan más bien "poco" desde la Universidad, sin embargo, los estudiantes "de ciencias" lo hacían incluso con menos frecuencia $(\mathrm{M}=2,13$. DT= 1,06) que los "de letras" $(\mathrm{M}=2,38 . \mathrm{Dt}=1,082)[\mathrm{t}(838)=-3,286 . \mathrm{p}<0,05]$. Por otra parte, el porcentaje de estudiantes "de ciencias" que incluía al perfil de la Universidad de Málaga entre sus contactos era significativamente inferior $(35,9 \%)$ al de los estudiantes "de letras" $(46,5 \%)\left[\chi^{2}(2, \mathrm{~N}=855)=10,826 . \mathrm{p}<0,05\right]$. Ambos grupos presentaban un porcentaje idéntico $(17 \%)$ de individuos que habían incluido a profesores universitarios entre sus contactos de las redes. Tampoco se hallaron diferencias significativas cuando esta pregunta se refería específicamente a Twitter.

Por último, aunque ambos colectivos afirmaron utilizar poco o muy poco las redes sociales para fines académicos, los resultados constataron que los estudiantes "de ciencias" hacen un uso significativamente inferior al de los estudiantes "de letras" en las 11 actividades académicas planteadas en el cuestionario. Las mayores diferencias se hallaron en la frecuencia con que usan las redes para "Hacer trabajos de clase" $[\mathrm{t}(841)=-6,289 . \mathrm{p}<0,05]$, "Informarse sobre qué se ha hecho en clase" [t(616,749) $=-6,004 \cdot \mathrm{p}<0,05]$, "Estar al día de lo que ocurre en la asignatura" $[\mathrm{t}(838)=-5,524$. $\mathrm{p}<0,05]$, "Solucionar dudas con otros estudiantes" $[\mathrm{t}(622,180)=-5,367 . \mathrm{p}<0,05]$ e "Intercambiar documentación y recursos útiles para la asignatura" $[\mathrm{t}(667,540)=$ $-5,328 . \mathrm{p}<0,05]$. 


\section{Conclusiones}

Esta investigación ha proporcionado un gran número de evidencias empíricas que permiten aproximar una respuesta a la pregunta de investigación que plantaba si existen diferencias en el uso académico de las redes sociales que hacen los universitarios de distintas áreas de conocimiento. A la luz de los datos descritos, podemos enunciar y discutir las siguientes conclusiones.

Los estudiantes "de letras" usan con mayor intensidad las redes sociales que los "de ciencias". El uso genérico de redes sociales en los dos colectivos estudiados es muy alto, sin embargo, los estudiantes "de ciencias" presentan una exposición y frecuencia de uso más reducida que sus compañeros "de letras". He aquí una paradoja: el peso de los estudiantes de cursos más bajos $\left(1^{\circ}\right.$ y $\left.2^{\circ}\right)$ en el conjunto del colectivo era superior entre el alumnado "de ciencias", lo que a priori debería haber generado unos datos de exposición y uso genérico superiores al de los estudiantes "de letras", colectivo integrado por un mayor número de alumnos de cursos superiores $\left(3^{\circ}\right.$ y $\left.4^{\circ}\right)$. Quizás la explicación a este resultado se deba a una mayor carga de trabajo y tiempo dedicado al estudio del alumno de las titulaciones "de ciencias". Viendo reducido su tiempo de ocio"- - no olvidemos que sigue siendo el primer motivo de uso de las redes-, frente al de sus compañeros "de letras", tendría sentido que el lapso destinado a las redes se redujera proporcionalmente tal y como demuestran los datos.

Por otra parte, los estudiantes "de letras" presentan en general una actitud más favorable que los "de ciencias" a la utilización de las redes sociales en la enseñanza universitaria. He aquí que nos encontramos de nuevo con el recurrente dilema sobre la relación entre actitudes y comportamiento. De un lado, podemos pensar que el mayor uso de las redes y, por tanto, de conocimiento que tienen los estudiantes "de letras" explica por qué sus actitudes son más favorables que las de sus compañeros "de ciencias". No obstante, podríamos enunciar la misma afirmación en sentido inverso. Es decir, el hecho de que los estudiantes "de letras" presenten actitudes más favorables al empleo de las redes en la docencia explicaría por qué las utilizan con mayor frecuencia que los estudiantes "de ciencias". En lo que atañe exclusivamente al ámbito actitudinal, podría deberse a que los estudiantes "de ciencias" perciben a sus profesores y a sus estudios como representantes de un modelo de educación más formal o tradicional, donde lo poco convencional, como la actividad académica que podría desarrollarse en las redes tendría poco o ningún valor real para sus profesores $\mathrm{y}$, en consecuencia, para su formación. No obstante, esta explicación ha de entenderse como una hipótesis de trabajo para futuras investigaciones. Por otra parte, llama la atención el escaso apoyo académico que encuentran ambos colectivos, especialmente los estudiantes "de ciencias", en las redes sociales. Este dato apunta claramente a la aún limitada actividad con fines docentes del profesorado en las redes sociales. Asimismo, indica el poco provecho que obtienen los docentes que están apostando por ello. En tal caso, sería preciso seguir incidiendo en la formación y en el desarrollo de experiencias experimentales. 
Finalmente, los estudiantes "de letras" emplean las redes sociales con fines académicos en mayor medida que sus compañeros "de ciencias". Es lógico que las diferencias halladas en el uso genérico de las redes tengan su reflejo en el uso académico que dispensan a las redes ambos grupos. En investigaciones anteriores (véase Gómez, Roses y Farias, 2012), ya advertimos que la escasa utilización académica de las redes - sin hacer distinciones por áreas de conocimiento - podía deberse a la apatía o inexperiencia del profesorado con estas tecnologías. No obstante, en el caso que nos ocupa, quizás podamos esgrimir alguna razón adicional que arroje luz sobre las diferencias encontradas. Es probable que en las titulaciones "de letras" se estén realizando más trabajos en grupo, dado que la mayor diferencia se halló en el uso de las redes para este menester. Los trabajos de grupo, en definitiva, el trabajo colaborativo, suscitan mayores necesidades de comunicación entre el alumnado. Quizás esto propicie mayor actividad y contacto inter pares a través de las redes en los estudiantes "de letras". Por otra parte, sería preciso indagar con metodología cualitativa si acaso existen distintas culturas estudiantiles dentro de las áreas de conocimiento cuyas dimensiones pudieran tener efecto en cómo los estudiantes interactúan entre sí. A priori, percibimos que existen titulaciones donde aflora un mayor espíritu competitivo, o una conciencia más individual o colectiva como estudiantes, por ejemplo. Cada grupo cultural podría, por consiguiente, experimentar unas necesidades y usos comunicativos distintos para con sus compañeros.

Por todo ello, no caben recetas universales en el uso docente de las redes. Es preciso estudiar con antelación cada grupo de estudiantes a fin de diseñar el método que consiga los mejores resultados de aprendizaje. En algunos casos, quizás esto pase por descartar las redes sociales si los docentes no somos capaces de atender a las expectativas creadas a nuestros estudiantes.

\section{Referencias bibliográficas}

ALEMAÑY MARTÍNEZ, C. (2009). "Redes sociales: una nueva vía para el aprendizaje". En: Cuadernos de Educación y Desarrollo, $\mathrm{n}^{\circ}$ 1. [15-07-2013] [Disponible en http://www.eumed.net/rev/ced/01/cam4.htm].

AVENDAÑO, P. et al. (2012). "La intención de uso educativo de las redes sociales en el ámbito universitario". En: Artículos de las Jornadas de Enseñanza de la Ingeniería, Año 2, vol. 1, p. 24-27. [10-09-2013] [Disponible en http://sicyt.scyt. rec.utn.edu.ar/sctyp/jein2012/articulos/articulojeinvol1.pdf\#page=24].

BATISTA, E. (2007): Lineamientos pedagógicos para la enseñanza y el aprendizaje. Colombia: Universidad Cooperativa de Colombia.

BUCKINGHAM, D.; BAUTISTA MARTÍNEZ-RODRÍGUEZ, J. (2013). “Jóvenes interactivos: nueva ciudadanía entre redes sociales y escenarios escolares". En: Revista Comunicar, $\mathrm{n}^{\circ}$ 40. [18-07-2013] [Disponible en http://www.revistacomunicar.com/index.php?contenido $=$ detalles \&numero $=40 \&$ articulo $=40-2013-02]$. 
CASTEÑEDA QUINTERO, L. (2010). Aprendizaje con redes sociales. Tejidos educativos para los nuevos entornos. Sevilla: Eduforma, Mad.

COBO ROMANÍ, C.; PARDO KUKLINSKI, H. (2007). Planeta Web 2.o. Inteligencia colectiva o medios fast food. Barcelona: Grup de Recerca d'Interaccions Digitals (Universidad de Vic).

DE HARO OLLÉ, J.-J. (2010). Redes sociales para la educación. Madrid: Anaya Multimedia.

DE MIGUEL, A. et al. (2012). "El Facebook para la docencia en ingeniería marítima”. En: Actas del Congreso Internacional de Docencia Universitaria e Innovación, celebrado en Barcelona en julio de 2012. [10-09-2013] [Disponible en http:// www.cidui.org/revistacidui12/index.php/cidui12/article/view/313].

DUART, J.-M.; SANGRÀ, A. (2005). Aprender en la virtualidad. Barcelona: Gedisa.

ESPUNY, C. et al. (2011). "Actitudes y expectativas del uso educativo de las redes sociales en los alumnos universitarios". En: Revista de Universidad y Sociedad del Conocimiento (RUSC), Vol. 8, n. ${ }^{\circ}$ 1, p. 171-185. [10-09-2013] [Disponible en http://rusc.uoc.edu/ojs/index.php/rusc/article/view/v8n1-espuny-gonzalez-lleixa-gisbert/v8n1-espuny-gonzalez-lleixa-gisbert].

FERNÁNDEZ, M.-R.; REVUELTA, F.-I.; SOSA, M.-J. (2012). "Redes sociales y microblogging: innovación didáctica en la formación superior". En Revista Latinoamericana de Tecnología Educativa, vol. 11, $\mathrm{n}^{\circ}$ 1. [10-09-2013] [Disponible en http://dialnet.unirioja.es/servlet/articulo?codigo $=4162581]$.

GARRIGÓS, I. et al. (2010). "La Influencia de las redes sociales en el aprendizaje colaborativo". En: JENUI 2010 XVI Jornadas de Enseñanza Universitaria de la Informática. Santiago de Compostela: Escola Técnica Superior d'Enxeñaría, p. 531-534. [10-09-2013] [Disponible en http://hdl.handle.net/2099/11859].

GÓMEZ AGUILAR, M.; ROSES, S.; FARIAS, P. (2012). "El uso académico de las redes sociales en universitarios". En: Comunicar, $\mathrm{n}^{\circ}$ 38. [4-09-2013] [Disponible en http://goo.gl/z60ESg].

KIESLINGER, B.; EHMS, K. (2010). “Autoorganización del proceso de aprendizaje”. En KIESLINGER, B.; FUMERO, A (coord.). Manual del uso del software social. Madrid: Win Win Consultores.

LÓPEZ ZAPICO, M.; TASCÓN, J. (2013). "El uso de Twitter como herramienta para la enseñanza universitaria en el ámbito de las ciencias sociales. Un estudio desde la Historia Económica". En: TESI, vol. 14, n² . [10-09-2013] [Disponible en: http://campus.usal.es/ revistas_trabajo/index.php/revistatesi/article/ view/10233/10667].

MARTÍNEZ, R.; CORZANA, F.; MILLÁN, J. (2013). "Experimentando con las redes sociales en la enseñanza universitaria en ciencias". En: Revista Eureka sobre Enseñanza y Divulgación de las Ciencias, vol. 10, no 3, p. 394-405. [10-09-2013] [Disponible en: http://rodin.uca.es:8081/xmlui/bitstream/ handle/10498/15445/6-393-MartinezCorzana.pdf?sequence=7].

MENDIGUREN, T.; MESO, K.; PÉREZ, J.-A. (2012). "El uso de las redes sociales como guía de autoaprendizaje en la Facultad de Comunicación de la UPV-EHV". 
En: Comunicación Social, no6, p.107-122. [10-09-2013] [Disponible en: http:// hdl.handle.net/11162/29588].

MESO, K.; PÉREZ, J.-A.; MENDIGUREN, T. (2011). "La implementación de las redes sociales en la enseñanza superior universitaria". En: Revista Tejuelo, $\mathrm{n}^{\circ} 12$.

PEÑA, K.; PÉREZ, M.; RONDÓN, E. (2010). "Redes sociales en Internet: reflexiones sobre sus posibilidades para el aprendizaje cooperativo y colaborativo". En: Revista de Teoría y Didáctica de las Ciencias Sociales, ${ }^{\circ}$ 16. [15-07-2013] [Disponible en: http://www.saber.ula.ve/bitstream/123456789/33627/1/articulo9. pdf].

SALINAS, J. (2009). "Innovación educativa y TIC en el ámbito universitario: Entornos institucionales, sociales y personales de aprendizaje". En: II Congreso Internacional de educación a distancia y TIC. Lima (Perú).

SUBIRES, P.; OLMEDO, S. (2013). "Universidad, sociedad y networking: perspectivas ante el uso de las redes sociales de perfil académico profesional". En: Estudios sobre el Mensaje Periodístico, vol. 19. [10-09-2013] [Disponible en: http://revistas.ucm.es/index.php/ESMP/article/view/42188].

TÚNEZ, M.; SIXTO, J. (2012). "Las redes sociales como entorno docente: Análisis del uso de Facebook en la docencia universitaria". En: Píxel-Bit, Revista de Medios y Educación, $\mathrm{n}^{\circ}$ 41, p. 77-92. [10-09-2013] [Disponible en: http://acdc. sav.us.es/pixelbit/images/stories/p41/06.pdf

\section{Los autores}

Sergio Roses es profesor en el Departamento de Periodismo de la Universidad de Málaga y consultor en Comunicación. Autor de una docena de artículos sobre la profesión periodística, la credibilidad de los medios y la docencia universitaria en Periodismo en revistas como Journalism Studies, Comunicación y Sociedad, Estudios sobre el Mensaje Periodístico, Revista Comunicar, Revista Latina de Comunicación Social o Revista Observatorio OBS, entre otras. Coautor de un capítulo en "The Global Journalist in the 21st Century", editado por Routledge y dirigido por David H. Weaver y Lars Wilnat, ha sido colaborador del Informe Anual de la Profesión Periodística entre 2006 y 2011.

Pedro Farias es profesor titular de Periodismo en la Universidad de Málaga donde es vicerrector de Comunicación y Proyección Internacional. Especialista en estudios sobre periodistas, sistema de medios y la formación del Periodismo, ha sido Director del Informe Anual de la Profesión Periodística entre 2006 y 2011 y director de numerosos proyectos de investigación y proyectos de innovación educativa. Autor de artículos científicos publicados en revistas como Comunicación y Sociedad, Revista Comunicar o Estudios sobre el Mensaje Periodístico, entre otras, también ha publicado capítulos de libro como "Journalists in Spain", incluido en "The Global Journalist in the 21 st Century", editado por Routledge. 
Marisol Gómez Aguilar es profesora de Periodismo en la Universidad de Málaga. Coordinadora del Informe Anual de la Profesión Periodística entre 2005 y 2011, ha participado en proyectos de investigación financiados por la Unión Europea, el Gobierno Español y el Gobierno Andaluz desde 2005 y en proyectos de innovación educativa centrados en la aplicación de las TIC en la docencia y en la adaptación de la docencia en Periodismo al bilingüismo. Entre sus líneas de investigación destacan el análisis de la profesión periodística, centrada en la situación profesional de la mujer y en la formación académica de los periodistas; así como el estudio de la aplicación de las nuevas tecnologías en la docencia. 